EPJ manuscript No.

(will be inserted by the editor)

\title{
Electronic Properties Close to Dirac Cone in Two-Dimensional Organic Conductor $\alpha$-(BEDT-TTF $)_{2} I_{3}$
}

\author{
Shinya Katayama ${ }^{1}$, Akito Kobayashi ${ }^{2}$, and Yoshikazu Suzumura ${ }^{1}$ \\ 1 Department of Physics, Nagoya University, Nagoya 464-8602, Japan \\ 2 Institute for Advanced Research, Nagoya University, Nagoya 464-8602, Japan \\ Received 17 October 2008 / Revised in final form 8 December 2008
}

\begin{abstract}
A zero-gap state (ZGS) has been found in a bulk system of two-dimensional organic conductor, $\alpha$-(BEDT-TTF $)_{2} \mathrm{I}_{3}$ salt which consists of four sites of donor molecules in a unit cell. In the present paper, the characteristic of the ZGS is analyzed in detail and the electronic properties are examined in the vicinity of the Dirac point where the conduction and valence bands degenerate to form the zero-gap. The eigenvectors of the energy band have four components of respective sites, where two of them correspond to inequivalent sites and the other two correspond to equivalent sites. It is shown that the former exhibits an exotic momentum dependence around the contact point and the latter shows almost a constant dependence. The density of states of each site close to the Dirac point is calculated to demonstrate the temperature dependence of the local magnetic susceptibility and the local nuclear magnetic relaxation rate. Further, the robust property of the ZGS against the anion potential is also shown by using the second-order perturbation.
\end{abstract}

PACS. 71.10.-w Theories and models of many-electron systems - 72.80.Le Polymers; organic compounds (including organic semiconductors)

\section{Introduction}

Organic conductor, BEDT-TTF

(bis(ethylene)dithiotetrathiafulvalene) salt has been studied extensively since the various kinds of the transfer energies between BEDT-TTF molecules show exotic phenomena in condensed matter physics 11. Recently, a quasi' two-dimensional conductor, $\alpha$-(BEDT-TTF) ${ }_{2} \mathrm{I}_{3}$ salt [2,3, becomes remarkable due to the theoretical finding of a massless Dirac particle under pressures [4,5] based on the experimental data of transfer energies [6]. Such a particle exhibits the the zero-gap state (ZGS) where the valence band and the conduction band touch each other at a momenta, $\pm \boldsymbol{k}_{0}$ in the Brillouin zone, and a Dirac point is produced. As the noticeable properties due to transfer energies, the location of $\boldsymbol{k}_{0}$ varies under pressure and the energy band around the Fermi energy is described by a linear dispersion but with an anisotropic Fermi velocity, i.e., by the tilted Weyl equation [7.

The intriguing property in the $\alpha$-(BEDT-TTF $)_{2} \mathrm{I}_{3}$ salt appears in the transport phenomena, which come from a zero-gap conductor. The Hall coefficient decreases rapidly with a power law as a function of temperature [8, while the electronic conductivity, $\sigma$, stays almost constant $(\sigma \sim$ $h / e^{2}$ per BEDT-TTF layer) [9]. Such a behavior of the transport property has been successfully explained in terms of the ZGS [10,11].
We note that there are several materials which show the Dirac fermions. The Dirac particle in condensed matter physics was first discovered in graphene (single layer graphite) [12,13. The graphene shows the isotropic linear dispersion around the corner of the Brillouin zone [14. [15. The structure of the Landau level 16,17, anomalous transport phenomena [18,19,20,21,22, and impurity effect $[23,24,25,26,27$, of the graphene have been proposed. Further, the bismuth has a small band gap with anisotropic velocities, and is described by the Dirac equation 28. The large diamagnetism appears due to the interband effect of magnetic field [29]. The ZGS also occurs in the HgTe quantum well by changing the thickness of the well [30.

There are still several issues on the ZGS of the $\alpha$ (BEDT-TTF $)_{2} \mathrm{I}_{3}$ salt, which are not yet clarified. (i) It is not obvious how the ZGS is constructed by BEDT-TTF molecules with several transfer energies. There are four molecules located at A, A', B, and C sites in a unit cell, where $\mathrm{A}$ and $\mathrm{A}^{\prime}$ are equivalent sites due to the inversion symmetry. The features of these three kinds of molecules (A, B, and C) are different with each other. There exists the charge disproportionation even at high temperatures [31,32, and the relation is found as $\left\langle n_{C}\right\rangle>\left\langle n_{A}\right\rangle=$ $\left\langle n_{A^{\prime}}\right\rangle>\left\langle n_{B}\right\rangle$ 33, where $\left\langle n_{\alpha}\right\rangle$ denotes the amount of the charge. The difference in the magnitude of $\left\langle n_{\alpha}\right\rangle$ becomes 
large under pressures 34. Further, it has been found recently that the local magnetic susceptibility $\chi_{\alpha}$ shows a relation, $\chi_{C}>\chi_{A}=\chi_{A^{\prime}}>\chi_{B}$ [34, which has the same relation as that of the charge disproportionation. In the present study, we examine the respective contribution of four different molecules to the ZGS, and show that the ZGS does not imply similarity between the susceptibilities and the charge disproportionation.

(ii) It is known that the ZGS is robust against pressure although the location of the contact point, $\pm \boldsymbol{k}_{0}$, varies due to variation of transfer energies under the pressure. Recently, the ZGS is examined in the presence of the anion potential, which gives the local potential on conduction electrons at each molecules 35. It is of interest to analyze the effect of the anion potential on the stability of the ZGS and the location of the contact point.

In the present paper, we study the role of the respective sites on the ZGS by calculating the local electronic state in the vicinity of the Dirac point. In $§ 2$, the energy bands and charge disproportionation are calculated for the $\alpha$ (BEDT-TTF $)_{2} \mathrm{I}_{3}$ salt by using transfer energies estimated from the first principle calculation. 36 The perturbation theory is also applied for the analysis of the electronic state close to the Dirac point and anion potential. In 93 the electronic state close to the Dirac point is calculated by focusing on the local properties of four donor molecules. In 44, the effect of the anion potential is examined. In 95 , the local density of states is calculated and is applied to the estimation of the temperature dependences of magnetic susceptibility and the nuclear magnetic relaxation rate. Finally, the summary is given in $\sqrt[6]{6}$.

\section{Band calculation}

The conducting layer of $\alpha$-(BEDT-TTF $)_{2} \mathrm{I}_{3}$ is shown in Figure 1, which are stacked with anion layers alternately. The notations of the bonds, $a 1, a 2, a 3, b 1, b 2, b 3$, and $b 4$ in Figure 1, are the same as those in Ref. [3], and quantities $a 1^{\prime}, a 3^{\prime}$, and $a 4^{\prime}$ denote those at next nearest neighbor sites, which were introduced by Kino and Miyazaki [36. In the present calculation, we adopt transfer energies evaluated from the first principle calculation at $T=8 \mathrm{~K}$ under the ambient pressure [36. Those in the unit of eV are given as $t_{a 1}=-0.0267, t_{a 2}=-0.0511, t_{a 3}=0.0323$, $t_{b 1}=0.1241, t_{b 2}=0.1296, t_{b 3}=0.0513, t_{b 4}=0.0152$, $t_{a 1^{\prime}}=0.0119, t_{a 3^{\prime}}=0.0046$, and $t_{a 4^{\prime}}=0.0060$. The unit of the energy is taken as $\mathrm{eV}$, hereafter. Other choice of transfer energies is discussed later.

The charge disproportionation in the $\alpha$-type BEDTTTF salt (Fig. 11) is examined by considering the extended

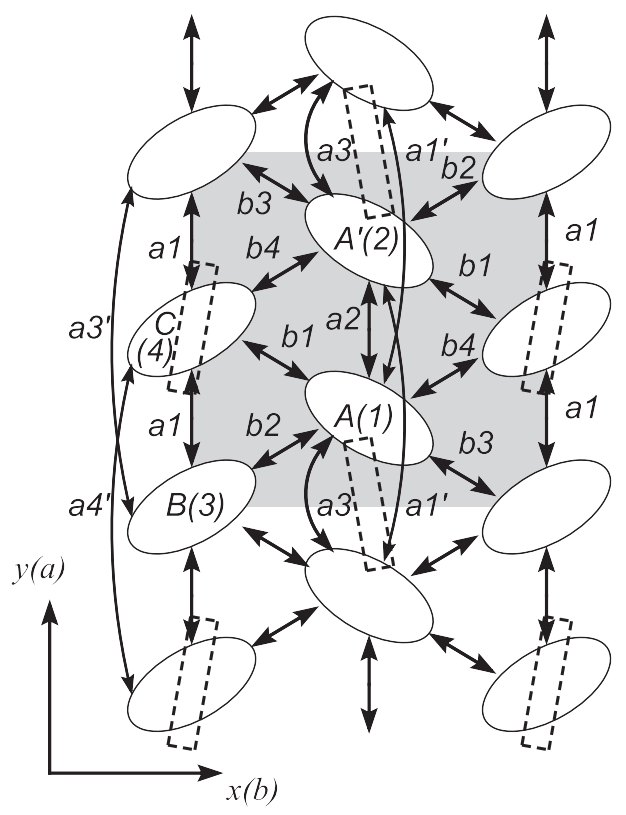

Fig. 1. Structure of the conducting plane of the $\alpha$-(BEDT$\mathrm{TTF})_{2} \mathrm{I}_{3}$ salt. The ellipse denotes a BEDT-TTF molecule and the unit cell of the salt is drawn by the gray square. The bonds, $a 1, a 2, a 3, b 1, b 2, b 3$, and $b 4$, denote the nearest neighbor electron hoppings and $a 1^{\prime}, a 3^{\prime}$, and $a 4^{\prime}$ denote the next nearest neighbor ones. Anions exist above and below dashed rectangles of conducting plane.

Hubbard model,

$$
\begin{aligned}
H & =\sum_{(i \alpha, j \beta), \sigma} t_{i \alpha: j \beta} c_{i \alpha \sigma}^{\dagger} c_{j \beta \sigma}+\sum_{i \alpha, \sigma} I_{\alpha} c_{i \alpha \sigma}^{\dagger} c_{i \alpha \sigma} \\
& +\sum_{i \alpha} U c_{i \alpha \uparrow}^{\dagger} c_{i \alpha \downarrow}^{\dagger} c_{i \alpha \downarrow} c_{i \alpha \uparrow} \\
& +\sum_{n . n ., \sigma \sigma^{\prime}} V_{i \alpha: j \beta} c_{i \alpha \sigma}^{\dagger} c_{j \beta \sigma^{\prime}}^{\dagger} c_{j \beta \sigma^{\prime}} c_{i \alpha \sigma},
\end{aligned}
$$

where $c_{i \alpha \sigma}$ denotes the annihilation operator of the fermion at the $\alpha$-th site in the $i$-th unit cell with the spin, $\sigma(=\uparrow, \downarrow)$. In Figure 1, sites $\mathrm{A}, \mathrm{A}, \mathrm{B}$, and $\mathrm{C}$ correspond to 1,2 , 3 , and 4 , respectively, and $\alpha, \beta=1,2,3$, and 4 . The quantity $t_{i \alpha: j \beta}$ is the transfer energy between nearest or next nearest sites. $U$ is the on-site Coulomb interaction. $V_{i \alpha: j \beta}$ is the off-site Coulomb interaction between $(i, \alpha)$ and $(j, \beta)$ sites, where the interaction is taken as $V_{a}$ for $a 1, a 2$, and $a 3$ bonds, and $V_{b}$ for $b 1, b 2, b 3$, and $b 4$ bonds. The local potentials at $\mathrm{A}\left(\mathrm{A}^{\prime}\right), \mathrm{B}$, and $\mathrm{C}$ sites are given by $I_{1}\left(=I_{2}\right), I_{3}$, and $I_{4}$, respectively, which are examined in ${ }_{4}$ The potential comes from the anion, $\mathrm{I}_{3}$, where the valence is given by -1 due to an electron transfer from two BEDT-TTF molecules. The anions also form a layer which is located between conducting layers. The position projected into the conducting plane (layer) is shown by dashed rectangles in Figure 1 [2]. 
We treat interactions within the mean-field approximation given by

$$
\begin{aligned}
& c_{i \alpha \sigma}^{\dagger} c_{j \beta \sigma^{\prime}}^{\dagger} c_{j \beta \sigma^{\prime}} c_{i \alpha \sigma} \\
& \rightarrow\left\langle n_{\alpha \sigma}\right\rangle c_{j \beta \sigma^{\prime}}^{\dagger} c_{j \beta \sigma^{\prime}}+c_{i \alpha \sigma}^{\dagger} c_{i \alpha \sigma}\left\langle n_{\beta \sigma^{\prime}}\right\rangle-\left\langle n_{\alpha \sigma}\right\rangle\left\langle n_{\beta \sigma^{\prime}}\right\rangle,
\end{aligned}
$$

where we take into account only Hartree terms and discard the exchange term. The quantity, $\left\langle c_{i \alpha \sigma}^{\dagger} c_{i \alpha \sigma}\right\rangle=\left\langle n_{\alpha \sigma}\right\rangle$ is calculated self-consistently. Using the Fourier transform, $c_{i \alpha \sigma}=(1 / \sqrt{N}) \sum_{\boldsymbol{k}} c_{\boldsymbol{k} \alpha \sigma} e^{\mathrm{i} \boldsymbol{k} \cdot \boldsymbol{r}_{i}}$, eq. (11) is rewritten as

$$
H_{M F}=\sum_{\boldsymbol{k}, \sigma}\left(c_{\boldsymbol{k} 1 \sigma}^{\dagger}, c_{\boldsymbol{k} 2 \sigma}^{\dagger}, c_{\boldsymbol{k} 3 \sigma}^{\dagger}, c_{\boldsymbol{k} 4 \sigma}^{\dagger}\right)(\hat{T}(\boldsymbol{k})+\hat{I})\left(\begin{array}{c}
c_{\boldsymbol{k} 1 \sigma} \\
c_{\boldsymbol{k} 2 \sigma} \\
c_{\boldsymbol{k} 3 \sigma} \\
c_{\boldsymbol{k} 4 \sigma}
\end{array}\right),
$$

where $\hat{T}(\boldsymbol{k})=\left[t_{\alpha \beta}(\boldsymbol{k})\right]$, and $\hat{I}=\left[I_{\alpha} \delta_{\alpha \beta}\right]$. The $4 \times 4$ matrix elements, $t_{\alpha \beta}(\boldsymbol{k})(\alpha, \beta=1, \cdots, 4)$, are given by

$$
\begin{array}{rlrl}
t_{11}(\boldsymbol{k}) & =2 t_{a 1^{\prime}} \cos k_{y}+C_{1}, & t_{12}(\boldsymbol{k})=t_{a 2}+t_{a 3} e^{-\mathrm{i} k_{y}} \\
t_{13}(\boldsymbol{k})=t_{b 2}+t_{b 3} e^{\mathrm{i} k_{x}}, & & t_{14}(\boldsymbol{k})=t_{b 1}+t_{b 4} e^{\mathrm{i} k_{x}} \\
t_{22}(\boldsymbol{k}) & =2 t_{a 1^{\prime}} \cos k_{y}+C_{2}, & & t_{23}(\boldsymbol{k})=t_{b 3} e^{\mathrm{i} k_{y}}+t_{b 2} e^{\mathrm{i}\left(k_{x}+k_{y}\right)} \\
t_{24}(\boldsymbol{k}) & =t_{b 4}+t_{b 1} e^{\mathrm{i} k_{x}}, & & t_{33}(\boldsymbol{k})=2 t_{a 3^{\prime}} \cos k_{y}+C_{3} \\
t_{34}(\boldsymbol{k}) & =t_{a 1}+t_{a 1} e^{-\mathrm{i} k_{y}}, & & t_{44}(\boldsymbol{k})=2 t_{a 4^{\prime}} \cos k_{y}+C_{4} \\
t_{\alpha \beta}(\boldsymbol{k}) & =t_{\beta \alpha}(\boldsymbol{k})^{*}, & &
\end{array}
$$

with

$$
\begin{aligned}
& C_{1}=U\left\langle n_{1}\right\rangle / 2+2 V_{a}\left\langle n_{2}\right\rangle+2 V_{b}\left(\left\langle n_{3}\right\rangle+\left\langle n_{4}\right\rangle\right), \\
& C_{2}=U\left\langle n_{2}\right\rangle / 2+2 V_{a}\left\langle n_{1}\right\rangle+2 V_{b}\left(\left\langle n_{3}\right\rangle+\left\langle n_{4}\right\rangle\right), \\
& C_{3}=U\left\langle n_{3}\right\rangle / 2+2 V_{a}\left\langle n_{4}\right\rangle+2 V_{b}\left(\left\langle n_{1}\right\rangle+\left\langle n_{2}\right\rangle\right), \\
& C_{4}=U\left\langle n_{4}\right\rangle / 2+2 V_{a}\left\langle n_{3}\right\rangle+2 V_{b}\left(\left\langle n_{1}\right\rangle+\left\langle n_{2}\right\rangle\right),
\end{aligned}
$$

where $\left\langle n_{\alpha}\right\rangle=\left\langle n_{\alpha \uparrow}\right\rangle+\left\langle n_{\alpha \downarrow}\right\rangle$. In eq. (5), the wave numbers $k_{x}$ and $k_{y}$ are measured by $1 / a$, where $a$ denotes a lattice constant. Note that, in the ZGS, there is no magnetic moment at any site i.e., $\left\langle m_{\alpha}\right\rangle\left(=\left\langle n_{\alpha \uparrow}\right\rangle-\left\langle n_{\alpha \downarrow}\right\rangle\right)=0$. The relation, $\left\langle n_{1}\right\rangle=\left\langle n_{2}\right\rangle$, is satisfied due to the inversion symmetry. Quantities $\left\langle n_{\alpha}\right\rangle$ and the chemical potential $\mu$ are calculated self-consistently by

$$
\begin{aligned}
& \sum_{\boldsymbol{k}}(\hat{T}(\boldsymbol{k})+\hat{I}-\mu \hat{E}) \boldsymbol{d}_{\gamma}(\boldsymbol{k})=\xi_{\gamma}(\boldsymbol{k}) \boldsymbol{d}_{\gamma}(\boldsymbol{k}), \\
& \left\langle n_{\alpha}\right\rangle=\frac{1}{N} \sum_{\boldsymbol{k} \gamma}\left|d_{\alpha \gamma}(\boldsymbol{k})\right|^{2} f\left(\xi_{\gamma}(\boldsymbol{k})\right), \\
& \sum_{\alpha}\left\langle n_{\alpha}\right\rangle=6,
\end{aligned}
$$

where $f(x)\left(=1 /\left(\mathrm{e}^{(x-\mu) / T}+1\right)\right)$ is the Fermi distribution function, and $T$ is a temperature (The unit of $T$ is given as $\mathrm{eV}$, and Boltzmann constant is taken as unity). For simplicity, we set $T=0$ and $f\left(\xi_{\gamma}(\boldsymbol{k})\right)$ in eq. (8) is replaced by a step function, $\theta\left(\varepsilon_{F}-\xi_{\gamma}(\boldsymbol{k})\right)$. $\hat{E}$ is the unit matrix. The $\alpha$-th component of the vector $\boldsymbol{d}_{\gamma}(\boldsymbol{k})(\alpha=1, \cdots, 4)$

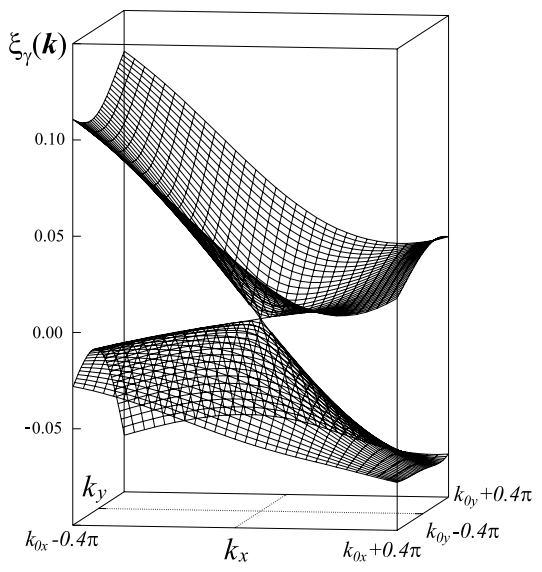

Fig. 2. Energy band, $\xi_{\gamma}(\boldsymbol{k})(\gamma=1,2)$, showing Dirac cone in the vicinity of the contact point, $\boldsymbol{k}_{0}=(0.686 \pi,-0.443 \pi)$. The Coulomb interactions, $U=0.4, V_{a}=0.17, V_{b}=0.05$, are taken into account in addition to the transfer energies, which are estimated by the first principle calculation at $8 \mathrm{~K}$ under ambient pressure. We set $\xi_{1}\left(\boldsymbol{k}_{0}\right)=\xi_{2}\left(\boldsymbol{k}_{0}\right)=0$.

is defined as $d_{\alpha \gamma}(\boldsymbol{k})$. The energy band, $\xi_{\gamma}(\boldsymbol{k})\left(\xi_{1}(\boldsymbol{k}) \geq\right.$ , $\left.\xi_{2}(\boldsymbol{k}) \geq \xi_{3}(\boldsymbol{k}) \geq \xi_{4}(\boldsymbol{k})\right)$, and the eigenvectors $\boldsymbol{d}_{\gamma}(\boldsymbol{k})$ are obtained by diagonalizing eq. (6).

We choose parameters, $U, V_{a}$, and $V_{b}$, in order to obtain the observed $\left\langle n_{\alpha}\right\rangle$ [33], in which the largest electron number is given at the $\mathrm{C}$ site and the smallest one is given at the B site. In the present calculation, these parameters are taken as $U=0.4, V_{a}=0.17, V_{b}=0.05$, and $I_{\alpha}=0.0$ 39, which give the ZGS with contact points, $\boldsymbol{k}_{0}= \pm(0.686 \pi,-0.443 \pi)$. Notice that the case $U=V_{a}=$ $V_{b}=0$ also gives the ZGS as shown in Ref. [36. For the present case, the energy band of the Dirac cone around the contact point is shown in Figure 2 The charge disproportionation is given by $\left\langle n_{1}\right\rangle=\left\langle n_{2}\right\rangle=1.460$ for $\mathrm{A}$ and $\mathrm{A}^{\prime}$ sites, $\left\langle n_{3}\right\rangle=1.369$ for $\mathrm{B}$ site, and $\left\langle n_{4}\right\rangle=1.711$ for $\mathrm{C}$ site.

Next, we examine the electronic state close to $\boldsymbol{k}_{0}$ within the perturbative method. Instead of the band index $\gamma$, we use $\zeta=1,2$ for valence and conduction bands and $\eta=3,4$ for the lower two bands. The Coulomb interaction and the charge disproportionation are determined unperturbative method, i.e., within eqs. (6)-(8). We construct the effective Hamiltonian as follows:

$$
H_{e f f}=H_{0}+V+V \frac{1}{E_{0}-H_{0}} V+\cdots,
$$

where $V$ is the perturbative Hamiltonian and the explicit form is given in the next section. $H_{0}$ denotes the Hamiltonian, eq. (3), at $\boldsymbol{k}_{0}$, with the effect of the charge disproportionation $\left(\left\langle n_{1}\right\rangle=\left\langle n_{2}\right\rangle=1.460,\left\langle n_{3}\right\rangle=1.369\right.$, $\left\langle n_{4}\right\rangle=1.711$, for $U=0.4, V_{a}=0.17, V_{b}=0.05$, and $\hat{I}=0)$. Since the system exhibits the ZGS, the upper two eigenvalues of $H_{0}$ degenerate on the Fermi energy, i.e. $E_{0}=\xi_{1}\left(\boldsymbol{k}_{0}\right)=\xi_{2}\left(\boldsymbol{k}_{0}\right)=0$, and the lower ones are given as $E_{3}=\xi_{3}\left(\boldsymbol{k}_{0}\right)=-0.218$, and $E_{4}=\xi_{4}\left(\boldsymbol{k}_{0}\right)=-0.404$. The eigenstates of $H_{0}$ described by the Luttinger-Kohn representation 37] for $\xi_{\zeta}\left(\boldsymbol{k}_{0}\right)$ and $\xi_{\eta}\left(\boldsymbol{k}_{0}\right)$ are given by $|\zeta\rangle$ 
$(\zeta=1,2)$ and $|\eta\rangle(\eta=3,4)$, respectively. Note that $|\zeta\rangle$ can not be determined uniquely due to the degeneracy. The detail forms of $\boldsymbol{d}_{1}$ and $\boldsymbol{d}_{2}$ are discussed in the next section. On the other hand, $|\eta\rangle$ can be described uniquely. Actually, the eigenvectors, $\boldsymbol{d}_{3}\left(\boldsymbol{k}_{0}\right)$ and $\boldsymbol{d}_{4}\left(\boldsymbol{k}_{0}\right)$ are calculated as

$$
\begin{aligned}
& \boldsymbol{d}_{3}\left(\boldsymbol{k}_{0}\right)=\left(\begin{array}{c}
0.5154 \\
-0.1944-0.4477 \mathrm{i} \\
-0.0944+0.4392 \mathrm{i} \\
-0.2410-0.4571 \mathrm{i}
\end{array}\right), \\
& \boldsymbol{d}_{4}\left(\boldsymbol{k}_{0}\right)=\left(\begin{array}{c}
0.5190 \\
0.3211+0.4077 \mathrm{i} \\
-0.4332-0.0311 \mathrm{i} \\
-0.4233+0.3057 \mathrm{i}
\end{array}\right) .
\end{aligned}
$$

When one of the linear combination of $|\zeta\rangle,\left|\psi_{\zeta}\right\rangle=a_{1 \zeta}(\boldsymbol{k})|1\rangle+$ $a_{2 \zeta}(\boldsymbol{k})|2\rangle$ is considered, we obtain the equation for the energy $\left(\Delta E_{\zeta}(\boldsymbol{k})\right)$ up to the second order of $V$ given as,

$$
\begin{aligned}
& \sum_{\zeta^{\prime \prime}=1,2}\left[\left\langle\zeta|V| \zeta^{\prime \prime}\right\rangle+\sum_{\eta=3,4} \frac{\langle\zeta|V| \eta\rangle\left\langle\eta|V| \zeta^{\prime \prime}\right\rangle}{\xi_{\eta}\left(\boldsymbol{k}_{0}\right)}\right] a_{\zeta^{\prime \prime} \zeta^{\prime}}(\boldsymbol{k}) \\
& =\Delta E_{\zeta}(\boldsymbol{k}) a_{\zeta \zeta^{\prime}}(\boldsymbol{k}) .
\end{aligned}
$$

In the next section, we discuss the ZGS using both the exact and perturbative calculations.

\section{Electronic state in the vicinity of the Dirac point}

In $₫ 3$ and $\$ 4$, the Dirac point $\boldsymbol{k}_{0}=(0.686 \pi,-0.443 \pi)$ is taken as the origin of the wave vector $\left(\boldsymbol{k}-\boldsymbol{k}_{0} \rightarrow \boldsymbol{k}\right)$.

First, we discuss the eigenvector with $\boldsymbol{k}$ close to $\boldsymbol{k}_{0}$. Figure 3 shows the $\theta$-dependences of $\left|d_{\alpha 1}(\theta)\right|\left(\right.$ a) and $\left|d_{\alpha 2}(\theta)\right|$ (b), which are calculated by diagonalizing eq. (8) $\left(\left(k_{x}, k_{y}\right)=\right.$ $\left.\delta^{\prime}(\cos \theta, \sin \theta)\right)$. The notable feature of Figure 3 (a) is the existence of a node for the components of both $\mathrm{B}$ site and $\mathrm{C}$ site, e.g., $d_{31}(0)=0$ and $d_{41}(\pi)=0$. For $\theta \sim 0$, $\left|d_{41}(\theta)\right|$ is the largest, whereas $\left|d_{31}(\theta)\right|$ becomes the largest for $\theta \sim \pi$. On the other hand, $\left|d_{\alpha 1}(\theta)\right|$ for $\alpha=1,2$ has weak $\theta$-dependence. It is also seen the relation, $\left|d_{\alpha 1}(\theta)\right|=$ $\left|d_{\alpha 2}(\theta+\pi)\right|$.

The behavior of Figure 3 is analyzed by the tilted Weyl equation. For small $|\boldsymbol{k}|$, the Hamiltonian, $V$, is written as

$$
V(\boldsymbol{k})=\left|\boldsymbol{k}_{0}, \alpha\right\rangle\left[\boldsymbol{k} \cdot \frac{\partial \hat{T}}{\partial \boldsymbol{k}}\left(\boldsymbol{k}_{0}\right)\right]\left\langle\boldsymbol{k}_{0}, \beta\right|,
$$

where $|\boldsymbol{k}, \alpha\rangle$ is the electronic state at site $\alpha(=1, \cdots, 4)$ with the wave number $\boldsymbol{k}$. By substituting eq. (12) into eq. (11) and discarding the second order term, the tilted Weyl (a)

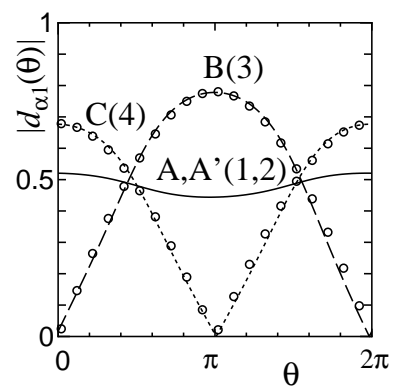

(b)

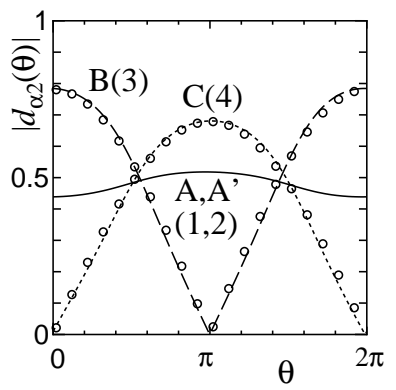

Fig. 3. $\theta$-dependence of the absolute values of the eigenvectors, $\left|d_{\alpha 1}(\theta)\right|(\mathrm{a})$ and $\left|d_{\alpha 2}(\theta)\right|(\mathrm{b})\left(\alpha=A, A^{\prime}, B, C\right)$. The open circles are obtained from eq. (21). The radius $\delta^{\prime}\left(=\left|\boldsymbol{k}-\boldsymbol{k}_{0}\right|\right)$ is set as $\delta^{\prime}=10^{-7} \pi$. $\theta$ denotes the angle between $\boldsymbol{k}-\boldsymbol{k}_{0}$ and $k_{x}$-axis.

equation is written as

$$
\begin{aligned}
& \boldsymbol{k} \cdot\left(\begin{array}{cc}
\tilde{\boldsymbol{v}}_{11} & \tilde{\boldsymbol{v}}_{12} \\
\tilde{\boldsymbol{v}}_{21} & \tilde{\boldsymbol{v}}_{22}
\end{array}\right)\left(\begin{array}{c}
a_{1 \zeta}(\boldsymbol{k}) \\
a_{2 \zeta}(\boldsymbol{k})
\end{array}\right) \\
& =\boldsymbol{k} \cdot\left(\begin{array}{cc}
\boldsymbol{v}_{0}+\boldsymbol{v}_{3} & \boldsymbol{v}_{1}-\mathrm{i} \boldsymbol{v}_{2} \\
\boldsymbol{v}_{1}+\mathrm{i} \boldsymbol{v}_{2} & \boldsymbol{v}_{0}-\boldsymbol{v}_{3}
\end{array}\right)\left(\begin{array}{c}
a_{1 \zeta}(\boldsymbol{k}) \\
a_{2 \zeta}(\boldsymbol{k})
\end{array}\right) \\
& =\sum_{\rho=0}^{3} \boldsymbol{k} \cdot \boldsymbol{v}_{\rho} \sigma_{\rho}\left(\begin{array}{c}
a_{1 \zeta}(\boldsymbol{k}) \\
a_{2 \zeta}(\boldsymbol{k})
\end{array}\right)=\Delta E_{\zeta}\left(\begin{array}{c}
a_{1 \zeta}(\boldsymbol{k}) \\
a_{2 \zeta}(\boldsymbol{k})
\end{array}\right) .
\end{aligned}
$$

The velocity, $\tilde{\boldsymbol{v}}_{\gamma \gamma^{\prime}}\left(\gamma, \gamma^{\prime}=1, \cdots, 4\right)$ is given by

$$
\tilde{\boldsymbol{v}}_{\gamma \gamma^{\prime}}=\sum_{\alpha \beta} \tilde{d}_{\alpha \gamma}^{*} \frac{\partial t_{\alpha \beta}}{\partial \boldsymbol{k}}\left(\boldsymbol{k}_{0}\right) \tilde{d}_{\beta \gamma^{\prime}},
$$

where $\tilde{d}_{\alpha \gamma}$ is defined as $\tilde{d}_{\alpha \zeta}=d_{\alpha \zeta}\left(\boldsymbol{k}_{0}^{\prime}\right)(\zeta=1,2)$ and $\tilde{d}_{\alpha \eta}=d_{\alpha \eta}\left(\boldsymbol{k}_{0}\right)(\eta=3,4)$. Since the components of the eigenvectors of the conduction $\left(d_{\alpha 1}\right)$ and valence $\left(d_{\alpha 2}\right)$ bands can not be determined at $\boldsymbol{k}_{0}$, the vector $\boldsymbol{k}_{0}^{\prime}=$ $\left(\delta^{\prime \prime} \cos \theta, \delta^{\prime \prime} \sin \theta\right)$ is introduced, where $\delta^{\prime \prime}$ is an infinitesimally small quantity and $\theta$ is the angle between $\boldsymbol{k}_{0}^{\prime}$ and $k_{x}$-axis. Velocities, $\boldsymbol{v}_{0}, \boldsymbol{v}_{1}, \boldsymbol{v}_{2}$, and $\boldsymbol{v}_{3}$, are expressed as $\boldsymbol{v}_{0}=\left(\tilde{\boldsymbol{v}}_{11}+\tilde{\boldsymbol{v}}_{22}\right) / 2, \boldsymbol{v}_{1}=\operatorname{Re}\left(\tilde{\boldsymbol{v}}_{12}\right), \boldsymbol{v}_{2}=-\operatorname{Im}\left(\tilde{\boldsymbol{v}}_{12}\right)$, and $\boldsymbol{v}_{3}=\left(\tilde{\boldsymbol{v}}_{11}-\tilde{\boldsymbol{v}}_{22}\right) / 2$, and $\sigma_{0}$ and $\sigma_{i}(i=1,2,3)$ denote unit matrix and Pauli matrix, respectively. The eigenvalue of eq. (13) is calculated as

$$
\begin{aligned}
& \Delta E_{1,2}(\boldsymbol{k})=\boldsymbol{k} \cdot \boldsymbol{v}_{0} \pm \sqrt{\sum_{\rho=1}^{3}\left(\boldsymbol{k} \cdot \boldsymbol{v}_{\rho}\right)^{2}} \\
= & v_{0 x} k_{x}+v_{0 y} k_{y} \pm \sqrt{V_{x}^{2} k_{x}^{2}+V_{y}^{2} k_{y}^{2}+2 V_{x y} k_{x} k_{y}},
\end{aligned}
$$

with $V_{x}^{2}=v_{1 x}^{2}+v_{2 x}^{2}+v_{3 x}^{2}, V_{y}^{2}=v_{1 y}^{2}+v_{2 y}^{2}+v_{3 y}^{2}$, and $V_{x y}=v_{1 x} v_{1 y}+v_{2 x} v_{2 y}+v_{3 x} v_{3 y}$. It should be noted that $\boldsymbol{v}_{1}$ and $\boldsymbol{v}_{2}$ depend on the wave number $\boldsymbol{k}_{0}^{\prime}$, whereas the velocity vectors $\boldsymbol{v}_{0}$ and $\boldsymbol{v}_{3}$ and quantities, $V_{x}^{2}, V_{y}^{2}$, and $V_{x y}$ are independent of the choice of the base. 

0, i.e.,

We choose $d_{\alpha \gamma}$ as the eigenvector of $t_{\alpha \beta}\left(\boldsymbol{k}_{0}\right)$ with $\theta=$

$$
\begin{aligned}
& \boldsymbol{x}_{1}=\boldsymbol{d}_{1}=\left(\begin{array}{c}
0.5194 \\
-0.4429+0.2713 \mathrm{i} \\
0 \\
0.6623+0.1473 \mathrm{i}
\end{array}\right), \\
& \boldsymbol{x}_{2}=\boldsymbol{d}_{2}=\left(\begin{array}{c}
0.4419 \\
0.3703-0.2411 \mathrm{i} \\
0.6125-0.4841 \mathrm{i} \\
0
\end{array}\right) .
\end{aligned}
$$

The velocity vectors are given as

$$
\begin{aligned}
& \boldsymbol{v}_{0}=(-0.0389,0.0048), \quad \boldsymbol{v}_{1}=(0,-0.0005), \\
& \boldsymbol{v}_{2}=(0,0.0439), \quad \boldsymbol{v}_{3}=(0.0515,0.0009)
\end{aligned}
$$

These vectors, are drawn in Figure 4. In this case, the effective Hamiltonian can be approximately written as

$$
H^{\prime}=\left(\begin{array}{cc}
v_{0 x} k_{x}+v_{0 y} k_{y}+v k_{x} & -\mathrm{i} v^{\prime} k_{y} \\
\mathrm{i} v^{\prime} k_{y} & v_{0 x} k_{x}+v_{0 y} k_{y}-v k_{x}
\end{array}\right),
$$

where $v_{0 x}=-0.0389, v_{0 y}=0.0048, v^{\prime}=v_{2 y}=0.0439$, and $v=v_{3 x}=0.0515$. Note that the effective Hamiltonian of eq. (18) differs from that of Ref. 10, since the choice of $\boldsymbol{k}_{0}^{\prime}$, i.e. the bases at $\boldsymbol{k}_{0}$, are not the same. However, the result does not depend on such a choice. The eigenvalue $\left(\Delta E_{\zeta}\right)$ and eigenvector $\left(a_{\zeta \zeta^{\prime}}\right)$ of eq. (18) are given as

$$
\begin{aligned}
& \Delta E_{1}=\omega=v_{0 x} k_{x}+v_{0 y} k_{y}+\sqrt{v^{2} k_{x}^{2}+v^{\prime 2} k_{y}^{2}} \\
& \Delta E_{2}=\omega^{\prime}=v_{0 x} k_{x}+v_{0 y} k_{y}-\sqrt{v^{2} k_{x}^{2}+v^{\prime 2} k_{y}^{2}}, \\
& \left(\begin{array}{c}
a_{11}(\theta) \\
a_{21}(\theta)
\end{array}\right)=\left(\begin{array}{c}
\cos (\theta / 2) \\
i \sin (\theta / 2)
\end{array}\right), \\
& \left(\begin{array}{l}
a_{12}(\theta) \\
a_{22}(\theta)
\end{array}\right)=\left(\begin{array}{c}
\sin (\theta / 2) \\
-\mathrm{i} \cos (\theta / 2)
\end{array}\right)
\end{aligned}
$$

where the relation $v \simeq v^{\prime}$ is used for the calculation of $a_{\zeta \zeta^{\prime}}(\boldsymbol{k})$, for simplicity. From eqs. (16) and (20), the $\theta$ dependence of $d_{\alpha \gamma}(\gamma=1,2)$ is obtained as

$$
\left(\begin{array}{l}
\boldsymbol{d}_{1}(\theta) \\
\boldsymbol{d}_{2}(\theta)
\end{array}\right)=\left(\begin{array}{ll}
a_{11}(\theta) & a_{12}(\theta) \\
a_{21}(\theta) & a_{22}(\theta)
\end{array}\right)\left(\begin{array}{l}
\boldsymbol{x}_{1} \\
\boldsymbol{x}_{2}
\end{array}\right)
$$

This gives $\left|d_{41}(\theta)\right|=\left|d_{32}(\theta)\right| \simeq 0.678|\cos (\theta / 2)|$ and $\left|d_{31}(\theta)\right|$ $\left|d_{42}(\theta)\right| \simeq 0.785|\sin (\theta / 2)|$ which reproduce well the exact one as shown in Figure 3 (open circle) within the accuracy of $\sim 0.03$.

Next, we discuss the detail structure of the Dirac cone. Equation (19) is rewritten as

$$
c_{1} k_{x}^{2}+c_{2} k_{y}^{2}+c_{3} k_{x} k_{y}+c_{4} k_{x}+c_{5} k_{y}=1
$$

where the coefficients are given by $c_{1}=\left(v^{2}-v_{0 x}^{2}\right) / \omega^{2}$, $c_{2}=\left(v^{\prime 2}-v_{0 y}^{2}\right) / \omega^{2}, c_{3}=2 v_{0 x} v_{0 y} / \omega^{2}, c_{4}=-2 v_{0 x} / \omega$ and $c_{5}=2 v_{0 y} / \omega$. Equation (22) represents the general form

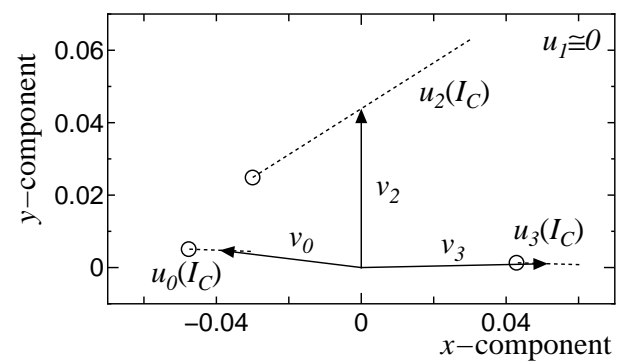

Fig. 4. Velocity vectors, $\boldsymbol{v}_{\rho}$ (eq. (17)) and $\boldsymbol{u}_{\rho}$ (eq. (30), where the bases are given by eq. (16). $\boldsymbol{v}_{\rho}$ are shown by arrows. For $I_{A}=I_{B}=0$, and $-0.2<I_{C}<0.2, \boldsymbol{u}_{\rho}(\rho=0,2,3)$ are shown by dotted line where open circles correspond to $I_{C}=0.2 . \boldsymbol{u}_{1}$ is small compared with other velocities $\left(\left|\boldsymbol{u}_{1}\right| \sim 10^{-3}\right)$.

of an ellipse as shown in Figure 5. The angle, $\phi$, between the long axis of the ellipse and $k_{x}$-axis, and the ratio, $a / b$, between the long and short axes are calculated as

$$
\begin{aligned}
\phi & =\frac{1}{2} \tan ^{-1} \frac{c_{3}}{c_{1}-c_{2}}, \\
\frac{a}{b} & =\sqrt{\frac{c_{1}+c_{2}+\sqrt{\left(c_{1}-c_{2}\right)^{2}+c_{3}^{2}}}{c_{1}+c_{2}-\sqrt{\left(c_{1}-c_{2}\right)^{2}+c_{3}^{2}}}}
\end{aligned}
$$

respectively. In the present case, one obtains $\phi<0$ and $a>b$. Further, by introducing a polar coordinate, $k_{x}=$ $r \cos \theta$ and $k_{y}=r \sin \theta$, eq. (22) is rewritten as

$$
r=\frac{\sqrt{4 A_{1}+A_{2}^{2}}-A_{2}}{2 A_{1}} \equiv F(\theta) \omega,
$$

where $A_{1}=c_{1} \cos ^{2} \theta+c_{2} \sin ^{2} \theta+c_{3} \cos \theta \sin \theta$ and $A_{2}=$ $c_{4} \cos \theta+c_{5} \sin \theta$. For $U=0.4, V_{a}=0.17, V_{b}=0.05$, the $\theta$-dependence of $F(\theta)$ is drawn in Figure 5 , where parameters are given as $a / \omega=48.00, b / \omega=35.20, X_{0} / \omega=36.09$, $Y_{0} / \omega=-7.082$, and $\phi=-0.089 \pi\left(\left(X_{0}, Y_{0}\right)\right.$ denotes the center coordinate of the ellipse.). The general form in terms of $X_{0}$ and $Y_{0}$ is shown in Appendix.

Let us compare the present result with that of the graphene. For the latter case, the Hamiltonian of eq. (13) is rewritten as $H^{\prime}=v\left(k_{x} \sigma_{1}+k_{y} \sigma_{2}\right)$, which denotes the isotropic Dirac cone. The absolute value of the component of the eigenvector is independent of $\theta$, i.e. $\left|d_{\alpha \gamma}(\theta)\right|=1 / \sqrt{2}$. The eigenvector of the latter case may correspond to the component of $\mathrm{A}$ and $\mathrm{A}^{\prime}$ of the former case, in which the $\theta$-dependence is small as shown in Figure 3. Thus it is found that the existence of the $\mathrm{B}$ and $\mathrm{C}$ components of $\overline{\bar{t}}$ the present case is the characteristic of the Dirac particle of organic conductor, $\alpha$-(BEDT-TTF $)_{2} \mathrm{I}_{3}$ salt, in which there are four sites in the unit cell. Further the vanishing of the $\mathrm{B}$ and $\mathrm{C}$ components at a certain value of $\theta$ seems to be general and exist for other case of transfer energies as discussed in $\$ 6$.

\section{The effect of the anion potential}

Next, we examine the anion potentials, $I_{A}\left(=I_{1}=I_{2}\right)$, $I_{B}\left(=I_{3}\right)$, and $I_{C}\left(=I_{4}\right)$ perturbatively. Although the charge 


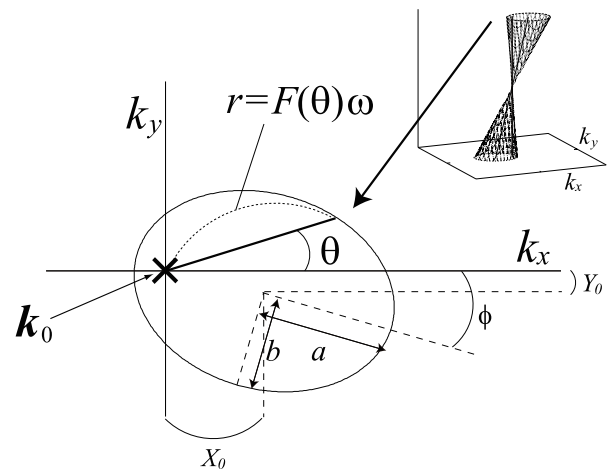

Fig. 5. Ellipsoidal curve obtained from the condition, $\xi=\omega$, for the Dirac cone. $\theta$ is the angle between $\boldsymbol{k}-\boldsymbol{k}_{0}$ and $k_{x}$-axis, where $\boldsymbol{k}_{0}$ is the point on the ellipsoidal curve. The inset denotes a tilted Dirac cone where $\omega=0$ corresponds to the apex, i.e., the Dirac point.

disproportionation depends on the anion potential 38, quantities of $\left\langle n_{\alpha}\right\rangle$ are fixed to $\left\langle n_{1}\right\rangle=\left\langle n_{2}\right\rangle=1.460,\left\langle n_{3}\right\rangle=$ 1.369 , and $\left\langle n_{4}\right\rangle=1.711$, which is obtained by the condition, $\hat{I}=0$. Such a treatment can be justified for the qualitative discussion of the location of $\boldsymbol{k}_{0}$ since the symmetry relation, $\left\langle n_{1}\right\rangle=\left\langle n_{2}\right\rangle$, remains due to $I_{1}=I_{2}$.

When the perturbative Hamiltonian is given as

$$
V(\boldsymbol{k})=\left|\boldsymbol{k}_{0}, \alpha\right\rangle\left[\boldsymbol{k} \cdot \frac{\partial \hat{T}}{\partial \boldsymbol{k}}\left(\boldsymbol{k}_{0}\right)+\hat{I}\right]\left\langle\boldsymbol{k}_{0}, \beta\right|,
$$

eq. (11) is rewritten as

$$
\sum_{\zeta^{\prime \prime}=1,2}\left[-\tilde{K}_{\zeta \zeta^{\prime \prime}}+\boldsymbol{k} \cdot \tilde{\boldsymbol{u}}_{\zeta \zeta^{\prime \prime}}+\tilde{J}_{\zeta \zeta^{\prime \prime}}\right] a_{\zeta^{\prime \prime} \zeta^{\prime}}(\boldsymbol{k})=\Delta E_{\zeta}(\boldsymbol{k}) a_{\zeta \zeta^{\prime}}(\boldsymbol{k})
$$

with

$$
\begin{gathered}
\tilde{K}_{\zeta \zeta^{\prime}}=\sum_{\eta=3,4} \frac{\left(\boldsymbol{k} \cdot \tilde{\boldsymbol{v}}_{\zeta \eta}\right)\left(\boldsymbol{k} \cdot \tilde{\boldsymbol{v}}_{\eta \zeta^{\prime}}\right)}{\xi_{\eta}\left(\boldsymbol{k}_{0}\right)} \\
\tilde{\boldsymbol{u}}_{\zeta \zeta^{\prime}}=\tilde{\boldsymbol{v}}_{\zeta \zeta^{\prime}}-\sum_{\eta=3,4} \frac{\tilde{\boldsymbol{v}}_{\zeta \eta} \tilde{I}_{\eta \zeta^{\prime}}+\tilde{I}_{\zeta \eta} \tilde{\boldsymbol{v}}_{\eta \zeta^{\prime}}}{\xi_{\eta}\left(\boldsymbol{k}_{0}\right)} \\
\tilde{J}_{\zeta \zeta^{\prime}}=\tilde{I}_{\zeta \zeta^{\prime}}-\sum_{\eta=3,4} \frac{\tilde{I}_{\zeta \eta} \tilde{I}_{\eta \zeta^{\prime}}}{\xi_{\eta}\left(\boldsymbol{k}_{0}\right)}
\end{gathered}
$$

where the matrix elements, $\tilde{I}_{\gamma \gamma^{\prime}}(\gamma=\zeta, \eta=1, \cdots, 4)$ is defined by

$$
\tilde{I}_{\gamma \gamma^{\prime}}=\sum_{\alpha} \tilde{d}_{\alpha \gamma}^{*} I_{\alpha} \tilde{d}_{\alpha \gamma^{\prime}}
$$

Discarding the first term of eq. (26) for simplicity, i.e. $\tilde{K}_{\gamma \gamma^{\prime}} \rightarrow 0$, the energy bands are calculated as

$$
\Delta E_{1,2}(\boldsymbol{k})=\boldsymbol{k} \cdot \boldsymbol{u}_{0}+J_{0} \pm \sqrt{\sum_{\rho=1}^{3}\left(\boldsymbol{k} \cdot \boldsymbol{u}_{\rho}+J_{\rho}\right)^{2}}
$$

Table 1. Quantities of coefficients, $K_{\rho x}^{\alpha}, K_{\rho y}^{\alpha}(\alpha=A, B, C$, $\rho=0,1,2,3)$.

\begin{tabular}{ccccc}
\hline$K_{\rho x}^{\alpha}$ & $\rho=0$ & $\rho=1$ & $\rho=2$ & $\rho=3$ \\
\hline $\mathrm{A}$ & -0.03 & 0.00 & 0.01 & 0.12 \\
$\mathrm{~B}$ & 0.08 & 0.00 & 0.14 & -0.07 \\
$\mathrm{C}$ & -0.04 & 0.00 & -0.15 & -0.04 \\
\hline \hline$K_{\rho y}^{\alpha}$ & $\rho=0$ & $\rho=1$ & $\rho=2$ & $\rho=3$ \\
\hline $\mathrm{A}$ & 0.01 & 0.00 & 0.07 & 0.00 \\
$\mathrm{~B}$ & 0.01 & 0.00 & 0.02 & 0.00 \\
$\mathrm{C}$ & 0.00 & 0.00 & -0.10 & 0.00 \\
\hline
\end{tabular}

where $\boldsymbol{u}_{0}=\left(\tilde{\boldsymbol{u}}_{11}+\tilde{\boldsymbol{u}}_{22}\right) / 2, \boldsymbol{u}_{1}=\operatorname{Re}\left(\tilde{\boldsymbol{u}}_{12}\right), \boldsymbol{u}_{2}=-\operatorname{Im}\left(\tilde{\boldsymbol{u}}_{12}\right)$, $\boldsymbol{u}_{3}=\left(\tilde{\boldsymbol{u}}_{11}-\tilde{\boldsymbol{u}}_{22}\right) / 2, J_{0}=\left(\tilde{J}_{11}+\tilde{J}_{22}\right) / 2, J_{1}=\operatorname{Re}\left(\tilde{J}_{12}\right)$, $J_{2}=-\operatorname{Im}\left(\tilde{J}_{12}\right)$, and $J_{3}=\left(\tilde{J}_{11}-\tilde{J}_{22}\right) / 2$. Compared with the previous section, the velocity is changed by the anion potential. Actually, for the bases chosen as eqs. (10) and (16), components of $\boldsymbol{u}_{\rho}(\rho=0, \cdots, 3)$ are given by

$$
\begin{aligned}
& u_{\rho x}=v_{\rho x}+K_{\rho x}^{A} I_{A}+K_{\rho x}^{B} I_{B}+K_{\rho x}^{C} I_{C}, \\
& u_{\rho y}=v_{\rho y}+K_{\rho y}^{A} I_{A}+K_{\rho y}^{B} I_{B}+K_{\rho y}^{C} I_{C}
\end{aligned}
$$

where coefficients of $K_{\rho x}^{\alpha}, K_{\rho y}^{\alpha}$ are given in Table 1. The velocity vector as a function of $I_{C}$ is drawn in Figure 4, where $-0.2<I_{C}<0.2$ and the other anion potentials are set as $I_{A}=I_{B}=0$. In the presence of $J_{\rho}$, the position of the contact point changes from that of the state with $\hat{I}=$ 0 . The quantities $J_{\rho}(\rho=0,1,2,3)$, which are expanded up to a quadratic term of the anion potential, are described by

$$
\begin{aligned}
J_{\rho}= & L_{\rho}^{A} I_{A}+L_{\rho}^{B} I_{B}+L_{\rho}^{C} I_{C}+L_{\rho}^{A A} I_{A}^{2}+L_{\rho}^{B B} I_{B}^{2}+L_{\rho}^{C C} I_{C}^{2} \\
& +L_{\rho}^{A B} I_{A} I_{B}+L_{\rho}^{C C} I_{B} I_{C}+L_{\rho}^{C A} I_{C} I_{A},
\end{aligned}
$$

where $L_{\rho}^{\alpha}$ is given in Table 2, The $I_{C}$-dependences of $J_{1}$, $J_{2}, J_{3}\left(I_{A}=I_{B}=0\right)$ are shown in the inset of Figure 6 . Note that $J_{1}$ and $J_{2}$ are negligibly small compared with $J_{3}$ (and also for case of the $I_{B}$-dependence with $I_{A}=I_{C}=$ $0)$. It should be noted that the effect of $J_{0}$ being the same order of $J_{3}$ does not contribute to the shape of the Dirac cone.

Using the above results, the energy difference between $\Delta E_{1}(\boldsymbol{k})$ and $\Delta E_{2}(\boldsymbol{k})$ is approximately given by

$$
\begin{aligned}
& \Delta E_{1}(\boldsymbol{k})-\Delta E_{2}(\boldsymbol{k}) \\
& \simeq 2 \sqrt{\left(u_{2 x} k_{x}+u_{2 y} k_{y}\right)^{2}+\left(u_{3 x} k_{x}+u_{3 y} k_{y}+J_{3}\right)^{2}} .
\end{aligned}
$$

Then, the contact point, which is given by $\Delta E_{1}-\Delta E_{2}=0$, is obtained at

$$
k_{0 x}=\frac{J_{3} u_{2 y}}{u_{2 x} u_{3 y}-u_{3 x} u_{2 y}}, \quad k_{0 y}=\frac{-J_{3} u_{2 x}}{u_{2 x} u_{3 y}-u_{3 x} u_{2 y}} .
$$

The trajectories of the contact point calculated from both eqs. (6) (circle) and (33) (line) are drawn in the main part 
Table 2. Quantities of coefficients, $L_{\rho}^{\alpha} \quad(\alpha=$ $A, B, C, A A, B B, C C, A B, B C, C A)$.

\begin{tabular}{ccccc}
\hline & $\rho=0$ & $\rho=1$ & $\rho=2$ & $\rho=3$ \\
\hline$L_{\rho}^{A}$ & 0.47 & 0.00 & -0.01 & 0.07 \\
$L_{\rho}^{B}$ & 0.30 & 0.00 & 0.01 & -0.30 \\
$L_{\rho}^{C}$ & 0.23 & 0.00 & 0.00 & 0.23 \\
\hline$L_{\rho}^{A A}$ & 0.86 & 0.00 & -0.26 & 0.01 \\
$L_{\rho}^{B B}$ & 0.42 & 0.00 & 0.01 & -0.42 \\
$L_{\rho}^{C C}$ & 0.44 & 0.00 & 0.00 & 0.44 \\
\hline$L_{\rho}^{A B}$ & -0.85 & 0.00 & 0.25 & 0.85 \\
$L_{\rho}^{B C}$ & 0.00 & 0.00 & -0.27 & 0.00 \\
$L_{\rho}^{C A}$ & -0.87 & 0.00 & 0.27 & -0.87 \\
\hline
\end{tabular}

of Figure 6. In case of the exact calculation, it moves from $\mathrm{Y}$ point to $\mathrm{M}$ point as shown in Ref. 38. The trajectory obtained from eq. (33) agrees qualitatively with that of eq. (6) for the small potential. Thus, one finds the ZGS is robust against the anion potential.

\section{Local density of states and magnetic properties}

By using the energy band and eigenvector (eq. (6) ), the density of states of the $\alpha$ site in a unit cell is calculated as

$$
D_{\alpha}(\varepsilon)=\frac{1}{N} \sum_{\boldsymbol{k} \gamma}\left|d_{\alpha \gamma}(\boldsymbol{k})\right|^{2} \delta\left(\varepsilon-\xi_{\gamma}(\boldsymbol{k})\right),
$$

where $\alpha=1(\mathrm{~A}), 2\left(\mathrm{~A}^{\prime}\right), 3(\mathrm{~B})$, and $4(\mathrm{C})$ denote the respective sites. The total value of the density of states is given by $\int \mathrm{d} \varepsilon \sum_{\alpha=1}^{4} D_{\alpha}(\varepsilon)=4$ and $\varepsilon=0$ denotes the chemical potential. Figure 7 depicts $D_{\alpha}(\varepsilon)$ close to the Fermi energy. The local density of states, $D_{\alpha}(\varepsilon)$, for small $\varepsilon$ is the largest at $\mathrm{C}$ site corresponding to the electron rich site, and is the smallest at B site corresponding to the hole (or charge) rich site [40. We notice that the above result is consistent with that of the experiment 33 .

The relative magnitude of $D_{\alpha}(\varepsilon)$ is understood as follows. From w3 we obtain that $d_{\alpha \gamma}(\boldsymbol{k})$ depends on only $\theta\left(=\tan ^{-1}\left[k_{y} / k_{x}\right]\right)$ in the vicinity of the Dirac point, and that the energy band is described by eq. (24). Then $D_{\alpha}(\varepsilon)$ $(\varepsilon>0)$ can be rewritten as

$$
\begin{aligned}
D_{\alpha}(\varepsilon) & =\frac{|\varepsilon|}{2 \pi^{2}} \int_{0}^{2 \pi} \mathrm{d} \theta \int_{0}^{\infty} \mathrm{d} r r\left|d_{\alpha 1}(\theta)\right|^{2} \delta(\varepsilon-r / F(\theta)) \\
& =\frac{|\varepsilon|}{2 \pi^{2}} \int_{0}^{2 \pi} \mathrm{d} \theta\left|d_{\alpha 1}(\theta)\right|^{2}(F(\theta))^{2}
\end{aligned}
$$

and

$$
\sum_{\alpha=1}^{4} D(\varepsilon)=\frac{|\varepsilon|}{2 \pi^{2}} \int_{0}^{2 \pi} \mathrm{d} \theta(F(\theta))^{2}
$$

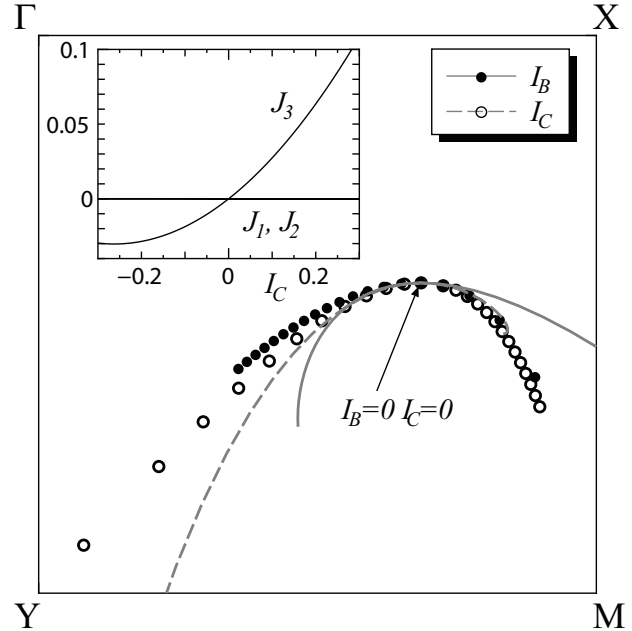

Fig. 6. Trajectory of the contact point in the quarter part of the Brillouin zone $\left(0<k_{x}<\pi,-\pi<k_{y}<0\right)$. Filled (open) circle denotes the position of $\boldsymbol{k}_{0}$ calculated from eq. (6) by the condition $I_{A}=0,-0.3<I_{B}<0.3, I_{C}=0\left(I_{A}=0, I_{B}=\right.$ $\left.0,-0.3<I_{C}<0.3\right)$. The corresponding result obtained from eq. (33) is drawn by solid (dashed) line. The inset shows the quantities $J_{1}, J_{2}$, and $J_{3}$ as functions of $I_{C}$.

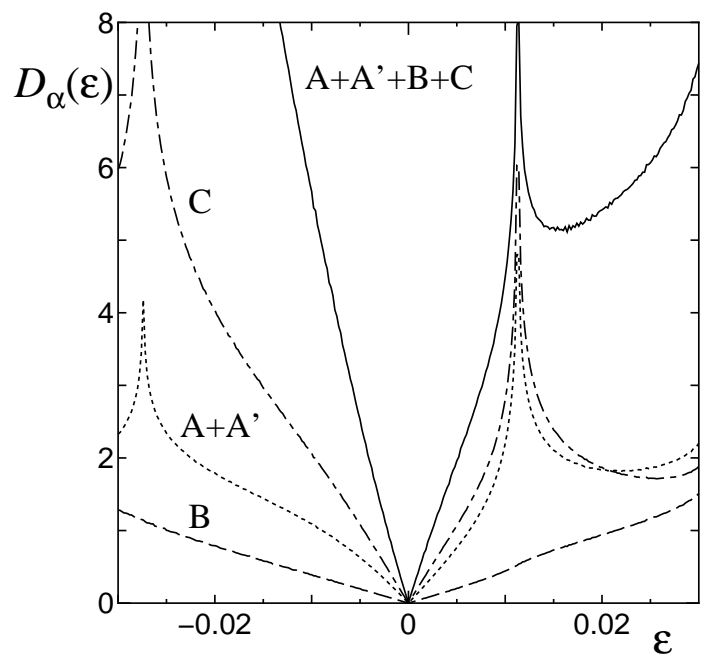

Fig. 7. Local density of states $D_{\alpha}(\varepsilon)$ per unit cell for A and A' sites (dotted line), B site (dashed line), and C site (dot-dashed line), which are calculated from eq. (34). Solid line is the total density of states. The parameters of interactions are taken as $U=0.4, V_{a}=0.17$ and $V_{b}=0.05$.

in the low energy region. The integration of eq. (35) is dominant for $\theta \sim 0$, since $F(0)$ is larger than $F(\pi)$ as shown in Figure 5 . Further, from Figure 3 (a), we see that the component of the eigenvector satisfies a condition $\left|d_{41}(0)\right|>\left|d_{11}(0)\right|=\left|d_{21}(0)\right|>\left|d_{31}(0)\right|$. These facts give a conclusion that the contribution of the $\mathrm{C}$ site (electron rich site) to the density of state is larger than that of the B site (hole rich site). It is also found, within the present scheme of eq. (18), that the density of states of 
$\varepsilon<0$ is the same as that of $\varepsilon>0$, from the symmetry of the energy band, $\Delta E_{1}(\boldsymbol{k})=-\Delta E_{2}(-\boldsymbol{k})$ (see eq. (15)). The underlying symmetry is due to the transformation property of the effective Hamiltonian under space inversion [17. We note that eq. (36) is the same as $|\varepsilon| /\left(2 \pi^{2} v_{F}^{* 2}\right)$ obtained in Ref. [17. The qunatity $v_{F}^{*}$ is the renormalized velocity, and is estimated as $v_{F}^{*}=0.0097$ for the choice of the present parameters where the corresponding four effective velocities in Ref. 17 are given as $w_{0 x}=-0.039$, $w_{0 y}=0.008, w_{x}=0.052$, and $w_{y}=0.044$. For small $|\varepsilon|$, the total density of states shown in Figure 7 coincides well with both eq. (36) and $|\varepsilon| /\left(2 \pi^{2} v_{F}^{* 2}\right)$.

Finally, the magnetic properties are examined. The susceptibility is calculated on the basis of four sites in a unit cell. The susceptibility corresponding to the response between the $\alpha$ site and the $\beta$ site is described as,

$$
\begin{aligned}
& \chi_{\alpha \beta}^{0}(\boldsymbol{q}, \omega)= \\
& -\frac{1}{N} \sum_{\boldsymbol{k} \gamma \gamma^{\prime}} d_{\alpha \gamma}(\boldsymbol{k}+\boldsymbol{q}) d_{\beta \gamma}^{*}(\boldsymbol{k}+\boldsymbol{q}) d_{\beta \gamma^{\prime}}(\boldsymbol{k}) d_{\alpha \gamma^{\prime}}^{*}(\boldsymbol{k}) \\
& \times \frac{f\left(\xi_{\gamma}(\boldsymbol{k}+\boldsymbol{q})\right)-f\left(\xi_{\gamma^{\prime}}(\boldsymbol{k})\right)}{\xi_{\gamma}(\boldsymbol{k}+\boldsymbol{q})-\xi_{\gamma^{\prime}}(\boldsymbol{k})-\omega-\mathrm{i} \delta},
\end{aligned}
$$

where $\mathrm{i} \delta(\delta>0)$ is a infinitesimally small imaginary part. Using equation (37), the magnetic responses of both the local susceptibility and the local NMR relaxation rate 41, 42. at the $\alpha$ site are obtained as

$$
\begin{aligned}
\chi_{\alpha} & =\sum_{\beta} \operatorname{Re}\left(\chi_{\alpha \beta}^{0}(\mathbf{0}, 0)\right)=\int_{-\infty}^{\infty} d \varepsilon D_{\alpha}(\varepsilon)\left(-\frac{\partial f}{\partial \varepsilon}\right) \\
\left(\frac{1}{T_{1}}\right)_{\alpha} & =T \lim _{\omega \rightarrow 0} \sum_{\boldsymbol{q}} \frac{\operatorname{Im}\left(\chi_{\alpha \alpha}^{0}(\boldsymbol{q}, \omega)\right)}{\omega} \\
& =\pi T \int_{-\infty}^{\infty} d \varepsilon\left(D_{\alpha}(\varepsilon)\right)^{2}\left(-\frac{\partial f}{\partial \varepsilon}\right)
\end{aligned}
$$

respectively.

The local magnetic susceptibility and the local NMR relaxation rate for each site are shown as a function of temperature in Figures 8 and 9, respectively. The susceptibility shows a relation $\chi_{C}>\chi_{A}=\chi_{A^{\prime}}>\chi_{B}$, which agrees qualitatively with that of the experiment [34. The open circles denotes the total one, which is calculated using the temperature dependence of the chemical potential and $\left\langle n_{\alpha}\right\rangle$ (eqs. (6) -(8) ) and the transfer energy given by

$$
t_{L}(T)=\frac{t_{L}(300 \mathrm{~K})-t_{L}(8 \mathrm{~K})}{292 \mathrm{~K}}(T-300 \mathrm{~K})+t_{L}(300 \mathrm{~K}) .
$$

The data of $t_{L}(300 \mathrm{~K})$ and $t_{L}(8 \mathrm{~K})(L=a 1, \cdots, a 3, b 1, \cdots, b 4$ $\left.a 1^{\prime}, a 3^{\prime}, a 4^{\prime}\right)$ are given in Ref. [36. The deviation is visible for $T>0.005 \mathrm{eV}$. In Figures 8 and 9, reflecting the density of states, $\chi_{4}$ and $\left(1 / T_{1}\right)_{4}(\mathrm{C}$ site $)$ are the largest among those of the four sites. It is found that, at low temperature, $\chi_{\alpha}$ and $\left(1 / T_{1}\right)_{\alpha}$ are proportional to $T$ and $T^{3}$, respectively, due to $D_{\alpha}(\varepsilon) \propto|\varepsilon|$. Actually, eqs. (38) and

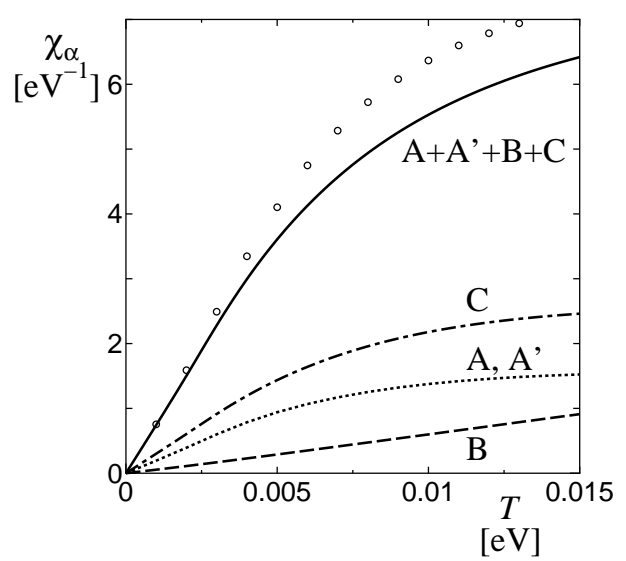

Fig. 8. Temperature dependence of $\chi_{\alpha}\left(\alpha=A, A^{\prime}, B, C\right)$. Dotted line, dashed line, and dot-dashed line correspond to A (A'), B, and C sites, respectively. The total value of $\chi_{\alpha}$ is plotted by the solid line where the open circle is calculated using eq. (40).

(39) at low temperature are calculated as $\chi_{\alpha}=2(\ln 2) K_{\alpha} T$ and $\left(1 / T_{1}\right)_{\alpha}=\left(\pi^{3} / 3\right) K_{\alpha}^{2} T^{3}$ where $D(\varepsilon)=K_{\alpha}|\varepsilon|$ from eq. (35)), where $K_{A} \simeq 137.1, K_{B} \simeq 38.9$ and $K_{C} \simeq 212.9$.

\section{Summary and discussion}

We examined the ZGS close to the Dirac point, and local magnetic properties at low temperature. Each electron in the unit cell has respective role to form a Dirac particle which is different from that of the graphene. The $\mathrm{C}$ and $\mathrm{B}$ sites give the largest and smallest density of states respectively, and are peculiar for the present salt $\alpha$-(BEDTTTF $)_{2} \mathrm{I}_{3}$ while the A and A' sites have a common feature with the graphene. The temperature dependence of magnetic susceptibility and NMR relaxation rate exhibit the power law at low temperature, which can be interpreted in terms of the density of states close to the Fermi surface.

Here we note that the detail of the ZGS depend on the choice of parameters as transfer energy, interaction and pressure. There are two kinds of conditions for the existence of the ZGS. First one is the contact point, which is satisfied for most of $\alpha$-type BEDT-TTF salt, and may arise from a property of an inversion symmetry of the four sites in the unit cell. The second one is that the contact point exists on the Fermi energy (i.e, the disappearance of hole or electron pockets). The ZGS has been obtained in the following cases. (i) Using the data of the X-ray experiment at room temperature [6] and $U=0.4, V_{a}=0.17$, $V_{a}=0.05$, the ZGS occurs for $P_{a}>4.3 \mathrm{kbar}$, whereas the 4,charge ordered insulating (metallic) state is obtained for 0 kbar $<P_{a}<3.3 \mathrm{kbar}\left(3.3 \mathrm{kbar}<P_{a}<4.3 \mathrm{kbar}\right)[4$. (ii) In the absence of interaction, the ZGS (metallic) state is obtained for $P_{a}>3 \mathrm{kbar}\left(3 \mathrm{kbar}<P_{a}\right)$ 5. (iii) For the small magnitude of interaction, the ZGS is obtained due to reducing the pockets while the large interaction destroys the ZGS due to the formation of the charge ordered 


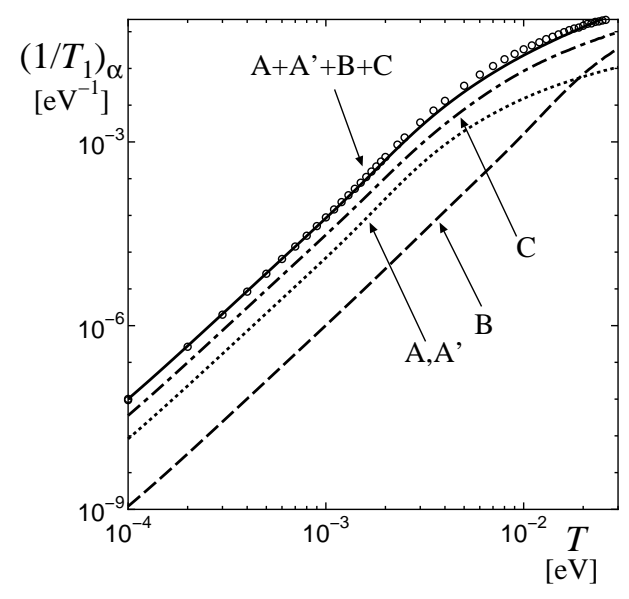

Fig. 9. Temperature dependence of $\left(1 / T_{1}\right)_{\alpha}$. Dotted line, dashed line, and dot-dashed line represent $\left(1 / T_{1}\right)_{\alpha}$ for $\mathrm{A}\left(\mathrm{A}^{\prime}\right)$, $\mathrm{B}$, and $\mathrm{C}$ sites, respectively. The total value of $\chi_{\alpha}$ is plotted by the solid line where the open circle is calculated by using eq. (40).

(CO) state. (iv) Further, the ZGS appears under higher hydrostatic pressure $(12.5 \mathrm{kbar}<P)$ [43].

We note the following relation between the charge disproportionation and the magnetic susceptibility in the ZGS Using parameters at $P_{a}=10 \mathrm{kbar}$ and room temperature [6], the charge disproportionation is given by $\left.\left\langle n_{C}\right\rangle\right\rangle$ $\left\langle n_{A}\right\rangle=\left\langle n_{A^{\prime}}\right\rangle>\left\langle n_{B}\right\rangle$ while the susceptibility $(\propto T)$ is obtained as $\chi_{A}\left(=\chi_{A^{\prime}}\right)>\chi_{C}>\chi_{B}$. This is contrast to the case for transfer energies calculated from the first principle calculation at $8 \mathrm{~K}$ and at ambient pressure [36], where $\left\langle n_{C}\right\rangle>\left\langle n_{A}\right\rangle=\left\langle n_{A^{\prime}}\right\rangle>\left\langle n_{B}\right\rangle$ and $\chi_{C}>\chi_{A}=\chi_{A^{\prime}}>\chi_{B}$. For the present choice of the transfer energy [36, the ZGS (CO) state is obtained for $x<1.03(x>1.03)$ with $U=0.4 x, V_{a}=0.17 x$ and $V_{b}=0.05 x$. Thus, we propose the following variation of interaction in order to explain the experiment that the $\mathrm{CO}$ state is obtained at ambient pressure and the ZGS is obtained for $P_{a}>4.3 \mathrm{kbar}$. The parameter of interaction at $P_{a}=0 \mathrm{kbar}$ is taken as $x>1.03$ and that of $P_{a}>4.3 \mathrm{kbar}$ is expected as $x<1.03$, since the effect of pressure increases the band width and decrease the relative magnitude of interactions.

Finally we discuss the state, which is derived from the transfer energies obtained from the experimental data at $T=8 \mathrm{~K}$ and at ambient pressure [6]. In this case, the ZGS emerges even at ambient pressure. Figure 10 shows the density of states under the ambient pressure where the parameters of interactions are the same as those of Figure 2. The charge disproportionation is obtained as $\left.\left.\left\langle n_{C}\right\rangle\right\rangle\left\langle n_{A}\right\rangle=\left\langle n_{A^{\prime}}\right\rangle\right\rangle\left\langle n_{B}\right\rangle$ which is qualitatively the same as the first principle calculation. 36 However, compared with Figure 7 the difference of each of $D_{\alpha}(\varepsilon)$ is very small in the vicinity of the Fermi energy. This implies that the similarity between $\chi_{\alpha}$ (corresponding to $D_{\alpha}(\varepsilon)$ ) and $\left\langle n_{\alpha}\right\rangle$ is not necessary for the existence of the ZGS. We also note that the node found in Figure 3 also exists in the case of Figure 10 although the location of the node is different. Thus the role of respective sites seems to be a common

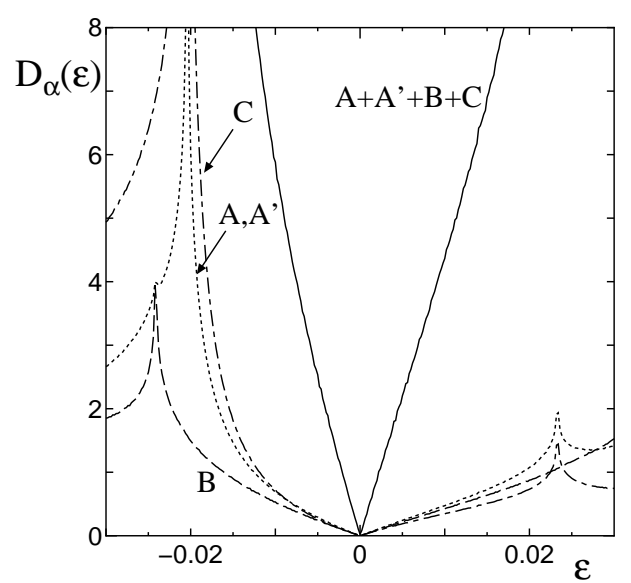

Fig. 10. Density of states at the ambient pressure and at $T=8 \mathrm{~K}$ where interactions are the same as Figure 7

feature for the ZGS of the system with four sites in the unit cell. Further we comment on a large Van Hove singularities, which exist for $\varepsilon>0$ in Figure 7 and for $\varepsilon<0$ in Figure 10. These singularities give opposite results for temperature effect, e.g. with increasing temperature, the chemical potential decreases for Figure 7 but increases for Figure 10 with increasing temperature. Thus the case of Figure 10 needs further consideration in order to explain a fact [10] that the behavior of the Hall coefficient suggests the monotonic decrease of chemical potential with increasing temperature.

We are grateful to Dr. Y. Takano and Prof. T. Takahashi for encouraging the motivation of our work and also for useful discussions. S. K. acknowledges the financial support of Research Fellowship for Young Scientists from Japan Society for the Promotion of Science (JSPS). This work was also financially supported by a Grant-in-Aid for Scientific Research on Priority Areas of Molecular Conductors (No. 15073103) from the Ministry of Education, Culture, Sports, Science and Technology, Japan.

\section{Appendix: A constant energy curve for the general tilted Weyl equation}

In this appendix, we examine the relation between the linear dispersion calculated from the tilted Weyl equation and the ellipse as shown in Figure 5. When we put $\lambda_{+}(\boldsymbol{k})=\omega$ in eq. (15), we obtain the general equation of the ellipse centered on $\left(X_{0}, Y_{0}\right)$ as follows:

$$
\begin{aligned}
& \frac{V_{x}^{2}-v_{0 x}^{2}}{R^{2}}\left(k_{x}-X_{0}\right)^{2}+\frac{V_{y}^{2}-v_{0 y}^{2}}{R^{2}}\left(k_{y}-Y_{0}\right)^{2} \\
& +\frac{V_{x y}^{2}-v_{0 x} v_{0 y}}{R^{2}}\left(k_{x}-X_{0}\right)\left(k_{y}-Y_{0}\right)=1,
\end{aligned}
$$


where

$$
R=\omega\left[\frac{V_{x}^{2} V_{y}^{2}-V_{x y}^{4}}{\left(V_{x}^{2}-v_{0 x}^{2}\right)\left(V_{y}^{2}-v_{0 y}^{2}\right)-\left(V_{x y}^{2}-v_{0 x} v_{0 y}\right)^{2}}\right]^{1 / 2} .
$$

The coordinates of the center $\left(X_{0}, Y_{0}\right)$ are given in the form:

$$
\begin{aligned}
& \frac{X_{0}}{\omega}=\frac{v_{0 y} V_{x y}^{2}-v_{0 x} V_{y}^{2}}{\left(V_{x}^{2}-v_{0 x}^{2}\right)\left(V_{y}^{2}-v_{0 y}^{2}\right)-\left(V_{x y}^{2}-v_{0 x} v_{0 y}\right)^{2}}, \\
& \frac{Y_{0 x} V_{x y}^{2}-v_{0 y} V_{x}^{2}}{\omega}=\frac{\left.v_{x y}^{2}-v_{0 x}^{2}\right)\left(V_{y}^{2}-v_{0 y}^{2}\right)-\left(V_{x y}^{2}-v_{0 x} v_{0 y}\right)^{2}}{\left(V_{x}^{2}\right.} .
\end{aligned}
$$

Carrying out the coordinate rotation,

$$
\begin{aligned}
& \left(\begin{array}{l}
k_{x}^{\prime} \\
k_{y}^{\prime}
\end{array}\right)=\left(\begin{array}{cc}
\cos \phi & \sin \phi \\
-\sin \phi \cos \phi
\end{array}\right)\left(\begin{array}{c}
k_{x}-X_{0} \\
k_{y}-Y_{0}
\end{array}\right) \\
& \phi=\left\{\begin{array}{c}
\frac{1}{2} \tan ^{-1}\left(\frac{2\left(V_{x y}^{2}-v_{0 x} v_{0 y}\right)}{\left(V_{x}^{2}-v_{0 x}^{2}\right)-\left(V_{y}^{2}-v_{0 y}^{2}\right)}\right) \\
\left(V_{x}^{2}-v_{0 x}^{2}<V_{y}^{2}-v_{0 y}^{2}\right) \\
-\frac{\pi}{2}+\frac{1}{2} \tan ^{-1}\left(\frac{2\left(V_{x y}^{2}-v_{0 x} v_{0 y}\right)}{\left(V_{x}^{2}-v_{0 x}^{2}\right)-\left(V_{y}^{2}-v_{0 y}^{2}\right)}\right) \\
\left(V_{x}^{2}-v_{0 x}^{2}>V_{y}^{2}-v_{0 y}^{2}\right)
\end{array}\right.
\end{aligned}
$$

we obtain the standard form of the ellipse (Fig. [5) given by

$$
\frac{k_{x}^{2}}{a^{2}}+\frac{k_{y}^{2}}{b^{2}}=1
$$

where the long and short axes are given as

$$
\begin{aligned}
a & =\frac{\sqrt{2} R}{\left[V_{x}^{2}-v_{0 x}^{2}+V_{y}^{2}-v_{0 y}^{2}-\sqrt{D}\right]^{1 / 2}}, \\
b & =\frac{\sqrt{2} R}{\left[V_{x}^{2}-v_{0 x}^{2}+V_{y}^{2}-v_{0 y}^{2}+\sqrt{D}\right]^{1 / 2}} \\
D & =\left[\left(V_{x}^{2}-V_{y}^{2}\right)-\left(v_{0 x}^{2}-v_{0 y}^{2}\right)\right]^{2}+4\left[V_{x y}^{2}-v_{0 x} v_{0 y}\right]^{2} .
\end{aligned}
$$

\section{References}

1. T. Ishiguro, K. Yamaji, and G. Saito: Organic Superconductors 2nd edn. (Springer-Verlag, Berlin, 1998)

2. K. Bender, I. Hennig, D. Schweitzer, K. Dietz, H. Endres, H. J. Keller, Mol. Cryst. Liq. Cryst. 108, 359 (1984)

3. T. Mori, A. Kobayashi, T. Sasaki, H. Kobayashi, G. Saito H. Inokuchi, Chem. Lett. 957 (1984)

4. A. Kobayashi, S. Katayama, K. Noguchi, Y. Suzumura, J. Phys. Soc. Jpn 73, 3135 (2004)

5. S. Katayama, A. Kobayashi, Y. Suzumura, J. Phys. Soc. Jpn 75, 054705 (2006)

6. R. Kondo, S. Kagoshima, J. Harada, Rev. Sci. Instrum. 76, 093902 (2005)
7. A. Kobayashi, S. Katayama, Y. Suzumura, H. Fukuyama, J. Phys. Soc. Jpn 76, 034711 (2007)

8. N. Tajima, M. Tamura, Y. Nishio, K. Kajita, Y. Iye, J. Phys. Soc. Jpn 69, 543 (2000)

9. N. Tajima, S. Sugawara, M. Tamura, R. Kato, Y. Nishio, K. Kajita, Euro. Phys. Lett. 80, 47002 (2007)

10. A. Kobayashi, Y. Suzumura, H. Fukuyama, J. Phys. Soc. Jpn 77, 064718 (2008)

11. S. Katayama, A. Kobayashi, Y. Suzumura, J. Phys. Soc. Jpn 75, 023708 (2006)

12. K. S. Novoselov, A. K. Geim, S. V. Morozov, D. Jiang, M. I. Katsnelson, I. V. Grigorieva, S. V. Dubonos, A. A. Firsov, Nature 438, 197 (2005)

13. Y. Zhang, Y. W. Tan, H. L. Stormer, P. Kim, Nature 438, $201(2005)$

14. P. R. Wallace, Phys. Rev. 71, 622 (1947)

15. H. Ajiki, T. Ando, J. Phys. Soc. Jpn 62, 1255 (1993)

16. T. Ando, J. Phys. Soc. Jpn 74, 777 (2005)

17. M. O. Goerbig, J. -N. Fuchs, G. Montambaux, F. Piechon, Phys. Rev. B 78, 045415 (2008)

18. T. Ando, T. Nakanishi, R. Saito, J. Phys. Soc. Jpn 67, 2857 (1998)

19. Y. Zheng, T. Ando, Phys. Rev. B 65, 245420 (2002)

20. V. P. Gusynin, S. G. Sharapov, Phys. Rev. B 73, 245411 (2006)

21. H. Fukuyama, J. Phys. Soc. Jpn 76, 043711 (2007)

22. K. Nomura, A. H. MacDonald: Phys. Rev. Lett. 98, 076602 (2007)

23. N. H. Shon, T. Ando, J. Phys. Soc. Jpn 67, 2421 (1998)

24. H. Kumazaki, D. S. Hirashima, J. Phys. Soc. Jpn 75, 053707 (2006)

25. N. M. R. Peres, F. Guinea, A. H. Castro Neto, Phys. Rev. B 73, 125411 (2006)

26. V. V. Cheianov, V. I. Fal'ko, Phys. Rev. Lett. 97, 226801 (2006)

27. T. O. Wehling, A. V. Balatsky, M. I. Katsnelson, A. I. Lichtenstein, K. Scharnberg, R. Wiesendanger, Phys. Rev. B 75, 125425 (2007)

28. P. A. Wolff, J. Phys. Chem. Solids 25, 1057 (1964)

29. H. Fukuyama, R. Kubo, J. Phys. Soc. Jpn 28, 570 (1970)

30. B. A. Bernevig, T. L. Hughes, S. C. Zhang, Science 314, 1757 (2006)

31. S. Moroto, K. Hiraki, Y. Takano, Y. Kubo, T. Takahashi, H. M. Yamamoto, T. Nakamura, J. Phys. IV France 114, 339 (2004)

32. Y. Takano, K. Hiraki, H. M. Yamamoto, T. Nakamura, T. Takahashi, Synth. Met. 120, 1081 (2001)

33. T. Kakiuchi, Y. Wakabayashi, H. Sawa, T. Takahashi, T. Nakamura, J. Phys. Soc. Jpn 76, 113702 (2007)

34. T. Takahashi, Y. Takano, private communication

35. R. Kondo, S. Kagoshima, N. Tajima, R. Kato, preprint

36. H. Kino, T. Miyazaki, J. Phys. Soc. Jpn 75, 034704 (2006)

37. J. M. Luttinger, W. Kohn, Phys. Rev. 97, 869 (1955)

38. S. Katayama, A. Kobayashi, Y. Suzumura, J. Phys. Conf. Series, 132, 012003 (2008)

39. A. Kobayashi, S. Katayama, Y. Suzumura: J. Phys. Soc. Jpn 74, 2897 (2005)

40. S. Komaba, Master Thesis, Nagoya University (2008).

41. T. Moriya, J. Phys. Soc. Jpn 18, 516 (1963)

42. Y. Suzumura, J. Phys. Soc. Jpn 58, 2642 (1989)

43. S. Katayama, A. Kobayashi, Y. Suzumura, J. Phys. Soc. Jpn 77, 014710 (2008) 